\title{
NO TODO LO QUE BRILLA ES ORO: ACCIÓN COLECTIVA EN MINERÍA AURÍFERA. BURITICÁ, ANTIOQUIA*
}

\author{
Juliana Toro ${ }^{a}$ \\ Julián Mazo-Zapata \\ Oswaldo Zapata
}

* DOI: https://doi.org/10.18601/01245996.v22n42.11. Este trabajo es parte de los proyectos "Gobernanza de minería aurífera" y "Contribución al estado de la cuestión para una teoría integrada de la acción colectiva”, Universidad Eafit. Recepción: 03-09-2018, modificación final: 13-09-2019, aceptación: 11-10-2019. Sugerencia de citación: Toro, J., Mazo-Zapata, J. y Zapata, O. (2020). No todo lo que brilla es oro: acción colectiva en minería aurífera. Buriticá, Antioquia. Revista de Economia Institucional, 22(42), 269-295.

a Magíster en Economía Aplicada. Cementos Argos, Medellín, Colombia, [jtorohe@eafit.edu.co], [https://orcid.org/0000-0002-9570-5374].

b Magíster en Economía Aplicada. Investigador asistente, Universidad Eafit, Medellín, Colombia, [jmazoza@eafit.edu.co], [https://orcid.org/00000001-7250-5532].

c Magíster en Economía. Consultor y docente, Universidad Eafit, Medellín, Colombia, [oswaldo.zapataq@gmail.com], [https://orcid.org/0000-00023788-2188]. 


\section{No todo lo que brilla es oro: acción colectiva en minería aurífera. El caso de Buriticá, Antioquia}

Resumen. Los territorios colombianos donde existe explotación minera han sido objeto numerosos estudios desde el punto de vista económico, político y social. En este artículo se analiza la acción colectiva en Buriticá, Antioquia, para entender sus dinámicas comunitarias a pesar de los retos de la explotación minera. Examina la cooperación a partir del mecanismo de contribución voluntaria y estima sus determinantes mediante un modelo probit. Los resultados indican que, aun en la adversidad, las personas tienden a actuar en forma cooperativa, a diferencia de estudios anteriores que se enfocan en la maximización del benefício individual.

Palabras clave: Acción colectiva, minería aurífera, experimentos económicos, prosocialidad; JEL: C90, D70, O17, P48

\section{All that glitters is not gold: collective action in gold mining.}

Abstract. The Colombian territories where mining occurs have been subject to numerous studies from an economic, political and social point of view. This article discusses the collective action in Buriticá, Antioquia, in order to understand its community dynamics, despite the challenges brought on by mining exploitation. It examines cooperation based on the voluntary contribution mechanism and estimates its determinants through a probit model. The results indicate that, even in adversity, individuals tend to act cooperatively, unlike previous studies that focus on maximizing individual benefits.

Keywords: Collective action, gold mining, economic experiments, prosociality; JEL: C90, D70, O17, P48

\section{Nem tudo o que reluz é ouro: ação coletiva na mineração de ouro. $O$ caso de Buriticá, Antioquia}

Resumo. Os territórios colombianos onde há mineração foram submetidos a numerosos estudos do ponto de vista econômico, político e social. Este artigo discute a ação coletiva em Buriticá, Antioquia, para entender sua dinâmica comunitária, apesar dos desafios da exploração mineira. Examina a cooperação com base no mecanismo de contribuição voluntária e estima seus determinantes por meio de um modelo probit. Os resultados indicam que, mesmo na adversidade, as pessoas tendem a agir cooperativamente, ao contrário de estudos anteriores que se concentram na maximização do benefício individual.

Palavras-chave: Ação coletiva, mineração de ouro, experimentos econômicos, prosocialidade; JEL: C90, D70, O17, P48 
T a minería ha sido una de las locomotoras del crecimiento ecoUnómico en los dos últimos gobiernos, y así lo refleja el hecho de que el sector extractivo es uno de los de mayor crecimiento. Aunque su estudio es relevante como tema económico, también es necesario entender el comportamiento de los agentes que participan directa o indirectamente en esa actividad.

La explotación aurífera es un tema de estudio reciente, desde el punto de vista económico, social y político, como ilustran los trabajos de Eslava et al. (2014), Rettberg et al. (2017), Rudas y Zamora (2017) y Valencia y Riaño (2017). Aunque estos trabajos muestran que el conflicto y la criminalidad son inherentes a la explotación del oro (Rettberg et al., 2017), también señalan que pese a la hostilidad existente en las zonas de explotación aurífera existen atributos comunitarios prosociales.

El proyecto minero de Buriticá es uno de los más grandes y ambiciosos del país, y abarca 56 ha en la vereda Higabra; aunque la compañía hoy tiene más de 40 solicitudes de títulos mineros en más de $30 \mathrm{mil}$ ha adicionales para nuevas exploraciones y posterior explotación, que según las proyecciones de la empresa duplicarían la producción legal de oro en el país (Continental Gold, 2017).

La llegada de una gran empresa, como Continental Gold, a Buriticá hace necesario entender los arreglos institucionales históricos del municipio para que la empresa no genere un desplazamiento que le cree dificultades a largo plazo. Es decir, que la actividad minera legal, con todas sus consecuencias, no destruya atributos comunitarios como la cooperación y la confianza.

Este artículo identifica los determinantes de la acción colectiva prosocial en Buriticá y hace recomendaciones de política para fortalecer ese capital social en la coyuntura actual. Para ello mide la disposición a la acción colectiva a través de juegos de laboratorio y explora su relación con algunos posibles factores explicativos de carácter individual y contextual. Con base en los resultados, sugiere cursos de acción -a empresarios, autoridades y comunidad-para proteger y fortalecer las inclinaciones prosociales a nivel local.

Se utiliza un método experimental (el mecanismo de contribución voluntaria, $\mathrm{MCV}$ ) que aporta evidencia empírica sobre la toma de decisiones y el comportamiento con respecto a otros, y es de uso común para establecer relaciones entre políticas públicas y gobernanza comunitaria, es decir, para determinar el nivel de acción colectiva. En el análisis de la acción colectiva es esencial entender los atributos prosociales para establecer relaciones armónicas entre los actores - 
Estado, agentes del mercado y comunidad-; que son necesarias para afrontar fallas de mercado como la información incompleta y los altos costos de transacción.

El artículo consta de seis secciones. En la primera se presenta el panorama de la minería aurífera en Buriticá como cuestión social y económica, y se da una justificación del trabajo. En la segunda se expone el marco teórico basado en los conceptos de acción colectiva prosocial y gobernanza comunitaria, así como la definición que se emplea en el trabajo. En la tercera se describe el procedimiento para obtener la información sobre acción colectiva y el método para estimar sus determinantes: el diseño experimental, la encuesta postjuego y los modelos de regresión. En la cuarta sección se presentan los resultados y en la última, las conclusiones.

\section{¿LA MINERÍA ES EL PROBLEMA?}

E1 sector extractivo es un tema de interés económico y social. Durante el periodo 2008-2013, este sector, que según el Dane corresponde a la explotación de minas y canteras, fue uno de los de mayor crecimiento en el país y el primer exportador y receptor de inversión extranjera directa (Pérez, 2017). El auge del sector estuvo ligado al alza de los precios del petróleo, el carbón y el oro, al crecimiento de economías emergentes, como las de China e India, y a la incertidumbre en los mercados internacionales. Y coincidió con la reducción de la tasa de cambio, que estimuló el crecimiento de las importaciones y las finanzas públicas. Según Pérez, el aporte del sector a estas últimas llegó a ser del 10\% anual. En suma, este sector fue una de las locomotoras del crecimiento económico del país, al menos hasta hace un par de años. Así lo confirman las cuentas nacionales, tanto en términos absolutos - por la riqueza y los ingresos crecientes que generó- como relativos - por su aporte cada vez mayor al PIB.

Como sugiere la gráfica 1, la explotación de minas y canteras ha hecho un gran aporte al crecimiento del país y de las regiones. Rudas y Espitia (2013) muestran que en el marco de la ley de garantías de 1994 (Ley 141) -por la cual las entidades territoriales productoras recibían la mayor parte de las ganancias que dejaba el sector-, los departamentos y municipios donde se concentraba el ingreso de esa actividad tuvieron un crecimiento sostenido ${ }^{1}$.

1 Aunque no mejoró la calidad de vida de sus habitantes. En los municipios donde se centró la producción de oro encontraron "heterogeneidad en relación a indicadores sociales e institucionales" (ibíd., 2013), como el índi- 
E1 departamento de Antioquia ha sido uno de los mayores extractores de oro y su producción ha aumentado más que el promedio nacional, y no obstante su crecimiento total ha sido inferior al promedio del país (Rudas y Espitia, 2013). Con la llegada de Continental Gold, se espera que la producción legal de oro aumente notablemente, lo que contribuiría al crecimiento del PIB nacional y departamental, y aumentaría el recaudo de impuestos y las regalías que reciben la nación y las entidades territoriales.

\section{Gráfica 1}

Minas y canteras, producción y participación en el PIB, 2005-2018

(Miles de millones de pesos y porcentaje)

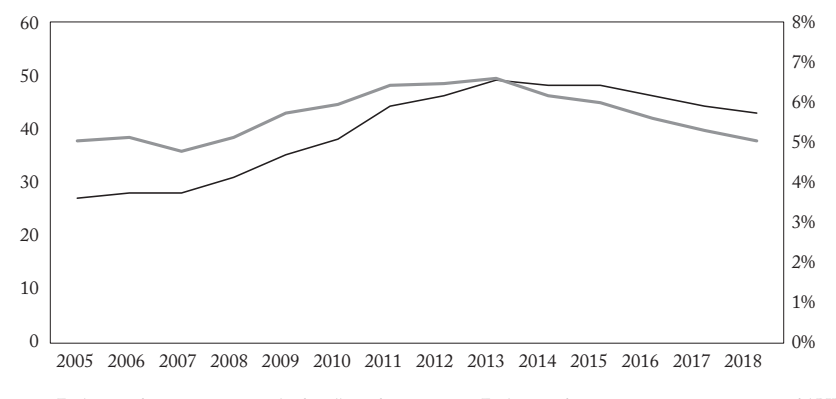

Fuente: Banco de la República (2019), elaboración propia.

Cabe destacar las tensiones causadas por la minería en Buriticá; desde la Colonia, cuando el cacique Buriticá prefirió morir que revelar a los colonos la ubicación del yacimiento de oro. La actividad se expandió bajo el empuje de María Zafra Centeno, quien empleó más de un centenar de esclavos.

Más tarde, en los años ochenta, llegaron extranjeros a extraer el metal y luego empresas como Grupo Bullet S.A., Centena S.A. y Continental Gold, la cual adquirió su primera licencia de explotación en 2007 (Múnera en Valencia y Riaño, 2017). Hoy, Continental Gold es el segundo grupo empresarial con mayor número de títulos mineros, después de Anglo Gold Ashanti (Cardona y Cuadros, 2014).

La llegada de Continental Gold al territorio riñe con diversos intereses, entre ellos los de las organizaciones criminales, que se lucran de la explotación ilegal de oro desde hace años. Y suscita inquietud la tensión que la empresa ha generado a nivel comunitario (Múnera en Valencia y Riaño, 2017), lo que es preocupante para la compañía, pues

ce multidimensional de condiciones de vida y la pobreza. Y eran más favorables en los municipios no mineros. 
en su planeación estratégica la comunidad es un actor fundamental en tanto receptora de los impactos de la explotación del metal, y como fuente histórica de gobernanza en el municipio.

\section{MARCOTEÓRICO}

E1 marco teórico relaciona tres categorías básicas - gobernanza comunitaria, acción colectiva y prosocialidad- con ayuda de cuatro categorías complementarias: instituciones, reglas, confianza y cooperación.

La acción colectiva se enmarca en la gobernanza comunitaria, entendida esta última como "arreglos de pequeñas interacciones sociales que junto con el mercado y el Estado determinan los resultados económicos" (Bowles y Gintis, 2005). Así, en la acción colectiva la comunidad se integra a la discusión de políticas públicas y se crea una gobernanza comunitaria junto con el Estado y el mercado. De modo que la gobernanza surge como resultado de la crisis de las formas tradicionales de gobierno (Subirats, 2010).

Para este autor catalán, el nuevo paradigma de la gobernanza reúne tres aspectos que constituyen su núcleo conceptual: el reconocimiento de la complejidad en el proceso político, la participación de diversos actores en redes plurales y la adopción de nuevos instrumentos de gobierno (ibíd.). A partir de ellos, define la gobernanza como "el escenario de interacción y cooperación de actores articulados en red para el desarrollo de proyectos colectivos" (íbid.) en los que la comunidad cobra importancia en cuanto genera confianza y cooperación, las cuales son necesarias para elaborar políticas públicas que respondan a la realidad local. Por medio de la gobernanza comunitaria se pueden enfrentar las fallas del Estado y del mercado porque la articulación de estos tres actores puede llevar a arreglos institucionales más eficientes.

Según Aguilar (2013), la gobernanza orienta y guía a una sociedad que reconoce la existencia de varios actores en un marco de interacción. La gobernanza comunitaria, tal como la definen Bowles y Gintis es una institución informal que complementa los arreglos institucionales formales -como el Estado y el mercado-, que si no tienen en cuenta los atributos de las comunidades desalientan sus inclinaciones prosociales. Para Charles Tilly y Leslie Wood (2010), las redes de confianza son fundamentales en la creación de las condiciones para establecer acuerdos formales o informales, puesto que cuando las comunidades se comprometen con arreglos institucionales o mercantiles se crea un escenario articulador que hace posible la trasmisión de información, y el cumplimiento y la supervisión de los compromisos (Eslava, 2017). 
Un resultado de las redes basadas en la confianza es la acción colectiva, que crea mecanismos para reducir la incertidumbre y los costos de transacción (Ostrom, 1990). La labor de la Continental Gold se facilitaría si se mantuviera y fortaleciera la acción colectiva existente en la zona, lo que no se puede dejar al curso espontáneo de los acontecimientos. Y como ya se mencionó, la imposición de reglas externas puede deteriorar los atributos de la comunidad, y menoscabar la confianza entre la empresa y la comunidad. Por ello es necesario identificar y comprender los determinantes de la acción colectiva en Buriticá, para diseñar arreglos institucionales que la fortalezcan y eviten una diáspora de los arreglos institucionales (institutional crowding-out) que perjudique el bienestar de la comunidad y las labores de la empresa.

En la concepción clásica de la acción colectiva algunos o todos los miembros de un grupo se dedican a la misma actividad (Elster, 1989). El problema de la acción colectiva se presenta cuando para todos es mejor que algunos o todos se dediquen a una misma actividad a que nadie se dedique a ella, pero para cada miembro es mejor no dedicarse a ella. Esta situación surge a partir de la incapacidad individual de alcanzar la máxima eficiencia del uso de un recurso, lo cual lo lleva a una coordinación voluntaria, es decir cooperativa, junto a otros individuos interesados en el consumo del bien y así evitar una situación de tragedia de los comunes.

Los estudios de Hardin (1968) y Olson (1965) abrieron el camino para una nueva generación de teorías de la acción colectiva, en la que se inscribe este trabajo. Uno de los grandes retos es proponer teorías consistentes que expliquen cómo un grupo de personas que enfrenta un dilema colectivo puede crear instituciones, comprometerse con ellas supervisarlas, de manera endógena (Ostrom, 1990). A diferencia de la primera generación, estas teorías, reconocen la multiplicidad de individuos y sus diversas características, y superan así la división dicotómica entre individuos cooperadores y no cooperadores. Entienden, entonces, que los individuos "difieren entre ellos en términos del grado en que se distancian de la motivación puramente egoísta" (Ostrom y Ahn, 2003, p. 180).

Dicha cooperación supone grados de confianza, que los participantes en la acción colectiva se forman expectativas mutuas acerca de sus acciones y que, a partir de ellas, actúan o no colectivamente. La expectativa sobre la acción del otro se traduce en reciprocidad cuando ese otro actúa de la manera esperada. 
Ostrom y Cárdenas (2004) analizan los incentivos individuales y las restricciones institucionales como guía de la cooperación, considerando el nivel de información que los individuos traen al juego, o a su contexto. Para estos autores, las instituciones -como reglas del juego- son esenciales en la toma de decisiones porque proporcionan información; sobre la identidad, el contexto del grupo y los incentivos o resultados del juego, "dependiendo del contexto que los individuos enfrentan, ellos pueden profundizar un poco más en un conjunto de niveles de información que es relevante para sus decisiones, por ejemplo, si el juego es llevadero, si la comunicación grupal es posible, y si los demás miembros y sus atributos son conocidos para los jugadores" (ibíd., p. 116).

En lo que respecta a la acción colectiva y el método experimental para estimar su magnitud, Cárdenas, Maya y López (2003) remiten al trabajo de Marwell y Ames (1981), el cual parte de la gran pregunta de Olson: ¿cuándo actúa una colectividad para maximizar el interés colectivo? Estos autores encuentran que, si bien los individuos actúan de manera oportunista en algunos casos, por lo general cooperan, y argumentan que su comportamiento real es distinto del que suelen suponer los economistas.

En cuanto a la prosocialidad, como ya se mencionó, la nueva generación de teorías subraya el papel de la comunidad y de sus atributos en la creación, el compromiso y la supervisión endógena de instituciones. Aspectos sobre los cuales, en opinión de Ostrom (1990), ni la teoría de la empresa ni la teoría del Estado dan explicación alguna. Bowles alude así a esos aspectos: "en contraste con los Estados y los mercados, las comunidades promueven y utilizan efectivamente los incentivos que la gente utiliza tradicionalmente para regular su actividad común: confianza, solidaridad, reciprocidad, reputación, orgullo personal, respeto, venganza y retribución, entre otros" (Bowles, 2010, p. 22). El adjetivo prosocial sugiere que el capital social es el factor más influyente en la cooperación voluntaria (Ostrom y Ahn, 2003).

\section{METODOLOGÍA}

\section{DISEÑO EXPERIMENTAL}

El instrumento para medir la acción colectiva (o disposición a cooperar) en Buriticá es un experimento denominado mecanismo de contribución voluntaria ${ }^{2}$, que da validez interna a la construcción

${ }^{2}$ Los procedimientos del experimento son una adaptación del diseño original (ver Méndez, 2012; Giraldo et al., 2013, y Eslava et al., 2014). 
teórica de los determinantes de la cooperación. Este método supera el obstáculo de la distancia entre preferencias declaradas y reveladas, y así ayuda a entender mejor la conducta humana y las decisiones de los individuos. En el experimento se observa la conducta de los individuos que emplean recursos monetarios reales en un ambiente donde el investigador controla el esquema de incentivos y la información disponible. En contra del supuesto tradicional de individuos racionales que maximizan sus beneficios, los experimentos revelan que si bien toman decisiones egoístas también toman decisiones que favorecen al grupo con base en la confianza y la cooperación; y que la acción colectiva resuelve dilemas sociales (Ostrom, 2013).

En los últimos diez años han cobrado importancia los métodos experimentales (Morton y Williams, 2010), debido al creciente interés por entender las relaciones causales, a las nuevas técnicas para analizar datos y a la aparición de nuevas preguntas difíciles de responder con los instrumentos tradicionales (Morton, 2013). La experimentación es un ejercicio de construcción de conocimiento basado en la generación de datos que ayudan a entender fenómenos sociales, aunque aún es objeto de debate, en particular sobre la validez de sus resultados.

Para Rebecca Morton y Kenneth Williams (2010), la validez -o aproximación a la verdad- del método experimental en ciencias sociales es un tema a amplio. Para delimitarlo, la dividen en validez interna y externa. Y, siguiendo a Cronbach (1982), descomponen la validez interna en validez estadística, replicabilidad estadística, validez causal y validez de los constructos (cuadro 1 ).

Cuadro 1

Componentes de la validación interna de los experimentos

\begin{tabular}{ll}
\hline $\begin{array}{c}\text { Componentes } \\
\text { de la validez interna }\end{array}$ & \multicolumn{1}{c}{ Descripción } \\
\hline Validez estadística & $\begin{array}{l}\text { Alude a la relación de covarianza entre las variables de interés y su } \\
\text { magnitud }\end{array}$ \\
\hline $\begin{array}{l}\text { Replicabilidad } \\
\text { estadística }\end{array}$ & $\begin{array}{l}\text { Consiste en la verificación de la validez: la posibilidad de replicar el } \\
\text { experimento y de que sus resultados tengan implicaciones teóricas } \\
\text { semejanzas }\end{array}$ \\
\hline Validez causal & $\begin{array}{l}\text { Alude a la posibilidad de determinar si las relaciones que el investiga- } \\
\text { dor encuentra son causales. Para Manski (1995), tiene dos dimensio- } \\
\text { nes: identificación de las relaciones y estimación de esas relaciones }\end{array}$ \\
\hline Validez de los & $\begin{array}{l}\text { Alude al vínculo que debe existir entre los conceptos teóricos en los } \\
\text { que se basa la pregunta que se busca responder y los hallazgos expe- } \\
\text { rimentales. Es decir, a la validez de las inferencias que se obtienen a } \\
\text { partir de los datos. "En la investigación experimental, la pregunta es si } \\
\text { el diseño de los experimentos es tal que las variables investigadas son } \\
\text { estrechamente equivalentes a las variables a las que se refiere la teoría” } \\
\text { (Morton y Williams, 2010) }\end{array}$ \\
\hline
\end{tabular}

Fuente: Morton y Williams (2010). 
La validez interna se refiere entonces a la capacidad para explicar el comportamiento de los individuos considerados. La validez externa, por su parte, se refiere a la posibilidad de generalizar los hallazgos a otros grupos: "si las inferencias causales establecidas en un análisis empírico se mantienen ante la variación de personas, entornos, variables de tratamiento y variables de medición” (Morton y Williams, 2006).

\section{VALIDEZ EXTERNA}

La validez externa, otro tema muy discutido, se refiere al poder de los experimentos y sus resultados para explicar el comportamiento de otras personas y grupos que no participan directamente en esos ejercicios, es decir, a la posibilidad de generalizar los resultados como si fuesen estimaciones de parámetros poblacionales.

Levitt y List (2007a) sostienen que los experimentos no son un espejo de la realidad, sino un espacio artificial en el que se recrean las instituciones que determinan la acción de los individuos. Los sujetos no se abstraen por completo del contexto social más amplio ni de sus experiencias y trayectorias de vida. La ética o los patrones de regulación social predominantes en un lugar determinado, así como la naturaleza y las preferencias de los jugadores, retroalimentan las decisiones aun en ese espacio controlado. Por ello, el investigador debe interpretar los resultados experimentales a la luz de la interacción entre las reglas propuestas en el juego y el contexto y los antecedentes de los participantes.

Uno de los propósitos más comunes de los experimentos es poner a prueba elaboraciones teóricas, y para ello basta una validez interna robusta y transparente (Schram, 2005). A diferencia de lo que ocurre en experimentos de campo, en experimentos de laboratorio la tensión entre validez externa y validez interna se resuelve en favor de la segunda, pues se privilegia el control que ejerce el investigador sobre las variables que pueden causar confusión más que el número de participantes o de decisiones.

Smith (1982) argumenta que en estos escenarios la validez externa no se refiere tanto a la posibilidad de generalizar los resultados como a la posibilidad de emplear el método en otros escenarios, lo que llama "paralelismo". Así, la validez externa se comprobaría en la medida que los hallazgos puedan ser corroborados o falseados en otros ambientes. "El paralelismo se define como la aplicabilidad de proposiciones que han sido probadas en el laboratorio a pequeñas economías donde la condición es similar [...], por lo que el paralelismo se refiere a la validez externa de los experimentos" (Levitt y List, 2007b). 
En suma, en este trabajo se sacrifica algo de la validez externa para lograr la mayor validez interna. Los resultados del experimento no son estadísticamente representativos de la población de Buriticá, pero sí suficientemente robustos para probar algunos postulados teóricos sobre la actitud prosocial en contextos de explotación minera. El diseño propuesto garantiza la validez externa, según el enfoque de Smith, pues ya existe un acervo de evidencia resultante de su empleo en el pasado, con el cual se pueden comparar nuestros resultados y, además, el procedimiento se puede replicar.

\section{MECANISMO DE CONTRIBUCIÓN VOLUNTARIA}

E1 diseño del experimento es simple; en el juego solo se toma una decisión: los participantes deciden si invierten su dotación inicial en una cuenta individual o en una cuenta grupal, e indican qué número participantes creen que la invertirán en la cuenta grupal.

Garrett Hardin y Mancur Olson fueron pioneros en el estudio de la acción colectiva. Si bien sus hallazgos son negativos de cara a su surgimiento y permanencia en el orden social, sentaron el punto de partida para otros trabajos, como los de Douglas North y Elinor Ostrom, donde la acción colectiva es una regla informal (North, 1993) o un modelo de gobernanza (Ostrom, 2013).

En 1965 se publicó La lógica de la acción colectiva de Mancur Olson, donde el autor examina la conducta de los grupos y, en particular, las dificultades de perseguir el bienestar común por encima del individual. Ese texto fue el punto de partida para desarrollos teóricos y metodológicos posteriores, entre ellos los juegos experimentales, el mecanismo de contribución voluntaria (o MCV) y sus variantes.

Para Saijo (2008) los principales hallazgos sobre la provisión de bienes públicos se han obtenido mediante el MCV. A partir de este juego se ha encontrado que, pese a que la estrategia dominante es no cooperar, los individuos cooperan. En opinión de Saijo, Ledyards (1995) y Andreoni (1995), el comportamiento irracional es más común de lo que se suele pensar y puede ser resultado de la confusión, la amabilidad o razones de justicia. Andreoni exploró esta tesis valiéndose del $\mathrm{MCV}$ para distinguir la motivación de la decisión, y encontró que gran parte de quienes contribuyeron a la cuenta grupal lo hicieron con el fin de cooperar.

Coleman y Ostrom (2009) muestran la pertinencia de este experimento y sostienen que los juegos experimentales -entre ellos el MCV- han contribuido al desarrollo de la teoría de la acción colectiva. 
En su opinión, ese método es indispensable para enfrentar la idea convencional del homo economicus.

Mucho se ha escrito sobre la acción colectiva. Estos estudios son particularmente útiles para los experimentadores porque proporcionan ejemplos de comportamientos y estrategias que las personas emplean en entornos reales para lograr la cooperación. Los experimentos han posibilitado estos comportamientos en entornos de laboratorio con el fin de evaluar exactamente qué tan efectivos pueden ser (Coleman y Ostrom, 2009, p. 4).

Ya se comentó la validez interna del diseño experimental. Y si bien es difícil generalizar los resultados, el presente trabajo identifica los determinantes de la acción colectiva revelada - no la declarada-entre actores clave del entorno minero de Buriticá, con un instrumento empleado en otros contextos y que, por ello, permite comparar los resultados, considerando variables empíricas con respaldo teórico (como propone Schram, 2005).

\section{ENCUESTA POSTJUEGO}

E1 diseño de esta encuesta se basa en la literatura sobre los determinantes de la acción colectiva y la cooperación, que sirve de guía para formular las preguntas más pertinentes y justificar la elección de las variables que se consideran determinantes. El cuadro 2 describe algunos de esos determinantes y los trabajos consultados. La encuesta postjuego se construyó con base en ellos y en preguntas que tuvieran continuidad con juegos anteriores.

Cabe resaltar algunos aspectos de estos determinantes. En experimentos donde los retornos y las decisiones varían según el tiempo, el riesgo y las consecuencias sociales se ha encontrado una relación de causalidad entre exposición a la violencia y victimización y aversión al riesgo. Los resultados estadísticos indican que la exposición a la violencia genera comportamientos altruistas en individuos, comunidades y en la sociedad, y aumenta la aversión al riesgo de los individuos, que dejan de esperar retornos de largo plazo pues no tienen la certeza de recibir el retorno. En suma, la violencia influye en las preferencias y en el comportamiento de los individuos. Aunque no es deseable, fomenta el altruismo y la cooperación (Voors et al., 2012).

Con respecto a la estatalidad local y la familiaridad, Dippel (2014) encontró que la acción colectiva depende de la confianza y de que el Estado la promueva. En ámbitos donde hay incoherencia entre lo institucional y lo social, es difícil que haya confianza y, por tanto, acción colectiva. Por su parte, Wolitzky (2012) argumenta que la cooperación que lleva a la acción colectiva surge de la coordinación 
Cuadro 2

Determinantes de la acción colectiva según la literatura empírica

\begin{tabular}{|c|c|c|}
\hline Determinante & Definición & Estudio \\
\hline Victimización & $\begin{array}{l}\text { Si durante su vida ha sido víctima de un } \\
\text { delito o crimen }\end{array}$ & \multirow{3}{*}{ Voors et al. (2012) } \\
\hline $\begin{array}{l}\text { Aversión al } \\
\text { riesgo }\end{array}$ & $\begin{array}{l}\text { Si decide teniendo en cuenta la incertidum- } \\
\text { bre sobre las consecuencias de las decisiones. }\end{array}$ & \\
\hline Familiaridad & $\begin{array}{l}\text { Aumento y disminución de la confianza } \\
\text { según el conocimiento, la intimidad y la } \\
\text { cercanía con el otro }\end{array}$ & \\
\hline Estatalidad local & $\begin{array}{l}\text { Percepción de la presencia del Estado en el } \\
\text { territorio }\end{array}$ & Dippel (2014) \\
\hline Expectativas & $\begin{array}{l}\text { Expectativas del individuo respecto a la ac- } \\
\text { ción futuro del otro y si le beneficiará o no }\end{array}$ & Wolitzky (2012) \\
\hline Información & $\begin{array}{l}\text { Calidad y cantidad de información que redu- } \\
\text { ce o aumenta los costos de transacción }\end{array}$ & Blume y Board (2013) \\
\hline Liderazgo & $\begin{array}{l}\text { Reconocimiento de figuras con atributos } \\
\text { comunitarios: capacidad de convocatoria, } \\
\text { ejemplo, vaso comunicante, etc. }\end{array}$ & Frey y Meier (2004) \\
\hline Arraigo & $\begin{array}{l}\text { Vínculo del individuo con el territorio por las } \\
\text { dinámicas sociales, culturales, económicas, o } \\
\text { políticas que crean valor agregado }\end{array}$ & Attanasio et al. (2015) \\
\hline Confianza & $\begin{array}{l}\text { Disposición a decidir y actuar en cierta } \\
\text { situación teniendo en cuenta la probabilidad } \\
\text { subjetiva del actuar de los demás }\end{array}$ & $\begin{array}{l}\text { Jackson, Rodriguez y Tan } \\
\text { (2012) }\end{array}$ \\
\hline Edad & Cuántos años tiene & \multirow{3}{*}{ Candelo y Polanía (2008) } \\
\hline Género & Es hombre o mujer & \\
\hline Educación & Años cursados en el sistema educativo & \\
\hline
\end{tabular}

Fuente: elaboración propia.

de expectativas. En su trabajo encontró que el conocimiento del grado de cooperación del otro y la supervisión horizontal promueven la acción colectiva.

En lo que se refiere a la información, Blume y Board (2013) encuentran que las asimetrías de información refuerzan los problemas de acción colectiva, por ejemplo, cuando hay distancia entre lo que saben y lo que se comunican; cuanto mayor es esa distancia menor es la acción colectiva. Finalmente, en cuanto a la confianza, Jackson et al. (2012) encuentran que la observación de las redes de favores y del capital social ayuda a detectar características que se correlacionan con el apoyo. La red informal y no coordinada de favores que funciona con base en la cercanía (amistades en común) es un mecanismo de acción colectiva que ayuda a establecer acuerdos que no logran el mercado ni el Estado. Estos autores argumentan que "gran parte del desarrollo, el crecimiento y el funcionamiento básico del día a día se basan en la capacidad de una sociedad para fomentar 'informalmente' el comportamiento cooperativo" (ibíd., p. 1857). 


\section{ESPECIFICACIÓN DEL MODELO}

En la notación siguiente se sigue a Wooldridge (2010). E1 modelo de regresión simple se especifica así:

$Y_{i}=\beta_{0}+\beta X_{i}+u_{i}$

donde $Y_{i}=0$ si la persona no aportó a la cuenta grupal y $Y_{i}=1$ si aportó a esa cuenta. El modelo se puede estimar de varias maneras. Aquí se emplean un modelo probabilidad lineal y dos modelos no lineales. Para el modelo lineal se tiene que:

$P(Y=1 \mid X)=G(X \beta)$

donde $G(X \beta)$ es la función de identidad; así, $P(Y=1 \mid X)=X \beta$.. $Y$ sigue una distribución de Bernoulli y el modelo es intrínsecamente heterocedástico. La probabilidad no está acotada entre 0 y 1 y los efectos marginales no dependen de los valores de otros regresores.

Para los modelos no lineales, que son de respuesta discreta, en el caso del modelo probit se tiene que:

$G(\mathrm{z})=\Phi(\mathrm{z})$, donde $\Phi(\mathrm{z})=\int_{-\infty}^{z} \phi(v) \mathrm{d} v \mathrm{y} \phi(v)$ es la distribución normal estándar.

En el caso del modelo logit se tiene:

$G(z)=\Lambda(z)$, donde $\Lambda(z)=\frac{\exp (z)}{1+\exp (z)}$

Para elegir el modelo se hicieron regresiones entre cada variable independiente y la variable dependiente-acción colectiva- para estimar la varianza explicada de la variable dependiente por cada variable independiente.

Después, se elaboró un modelo base con las regresiones que tenían el $\mathrm{R}^{2}$ más alto y eran, por tanto, más significativas. Luego se agregaron algunas variables que, según la literatura, es necesario incluir en el modelo. Los regresores finales fueron:

Pocoestado: percepción de que la poca presencia del Estado es o no uno de los tres principales problemas de Buriticá.

Ptajeexpect: promedio del número de jugadores que según el grupo aportarían a la cuenta grupal.

Benefgrupo: el individuo tomó o no decisiones en el MCV considerando el beneficio del grupo.

Cgold: la persona tiene nada, poca, algo o mucha confianza en Continental Gold.

Estadonosirve: la razón para que la persona participe en una organización social es o no la inoperancia del Estado. 
Despforzado: la persona ha sufrido o no de desplazamiento forzado.

Decaridad: la persona participa en una organización de caridad.

\section{ESTADÍSTICAS DESCRIPTIVAS}

En el municipio de Buriticá existen grandes retos relacionados con la gobernanza, por las disputas existentes entre el Estado, el mercado (representado por la compañía minera) y la comunidad. Es entonces un escenario ideal para identificar y reforzar los atributos prosociales con el fin de que hagan parte de la nueva planeación ante la llegada de un nuevo actor.

A continuación se presentan las estadísticas descriptivas, agregadas y desagregadas de cada grupo, con los datos recogidos en el MCV y en la encuesta postjuego. Se realizaron cuatro ejercicios con grupos representativos del municipio: misceláneo, mineros en proceso de formalización, miembros de organizaciones sociales de la vereda Los Asientos y funcionarios púbicos locales. Al final se obtuvieron datos de 71 participantes; el 43,7\% hombres y el 56,3\% mujeres, con rangos de edad de 18 a 30 años y de 31 a 50; y un promedio de edad de 37 años.

Cuadro 3

Rangos de edad

\begin{tabular}{lcccc}
\hline & $0-8$ & $18-30$ & $31-50$ & 51 y más \\
\hline Misceláneo & 0 & 8 & 6 & 4 \\
Mineros & 0 & 8 & 8 & 3 \\
Los Asientos & 2 & 4 & 7 & 5 \\
Funcionarios & 0 & 6 & 9 & 1 \\
\hline
\end{tabular}

Fuente: elaboración propia.

En el ejercicio cuyos participantes reportaron menos años de estudio fue el de la vereda Los Asientos, cuyo valor máximo (18 años) fue reportado por un funcionario de la empresa minera presente en el municipio. $\mathrm{Y}$ el ejercicio cuyos participantes reportaron un mayor número promedio de estudios fue el de funcionarios públicos, 14 años en promedio. Por otra parte, en el ejercicio cuyos participantes llevan viviendo mayor número de años en el municipio, fue el misceláneo, y el de menor número de años fue el de mineros (cuadro 4). 
Cuadro 4

Escolaridad y años de vida en Buriticá

\begin{tabular}{lccc}
\hline & Años promedio & Mínimo & Máximo \\
\hline Escolaridad & 10 & 0 & \\
Misceláneo & 12 & 5 & 16 \\
Mineros & 7 & 0 & 16 \\
Los Asientos & 14 & 6 & 18 \\
Funcionarios & & & 18 \\
Años de vida en Buriticá & 28 & 1 & \\
Misceláneo & 17 & 1 & 65 \\
Mineros & 23 & 0,3 & 75 \\
Los Asientos & 18 & 0,01 & 53 \\
Funcionarios &
\end{tabular}

Fuente: elaboración propia.

E1 cuadro 5 presenta los resultados agregados y el cuadro 6, las estadísticas descriptivas de las variables incluidas en los ejercicios econométricos.

Cuadro 5

Edad, escolaridad y arraigo

\begin{tabular}{lccc}
\hline & Edad & Escolaridad & $\begin{array}{c}\text { Arraigo } \\
\text { (años en el municipio })\end{array}$ \\
\hline Media & 37,05633803 & 10,61971831 & 24,62528571 \\
Mediana & 33 & 11 & 24 \\
Moda & 30 & 11 & 1 \\
Desviación estándar & 14,41119339 & 5,003901898 & 18,79904289 \\
Mínimo & 14 & 0 & 0,01 \\
Máximo & 77 & 18 & 75 \\
Observaciones & 71 & 71 & 70 \\
\hline
\end{tabular}

Fuente: elaboración propia.

Cuadro 6

Estadística descriptiva de las variables incluidas en los ejercicios econométricos

\begin{tabular}{|c|c|c|c|c|c|c|c|c|c|}
\hline & 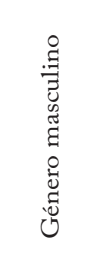 & 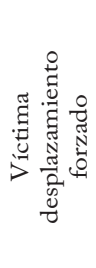 & 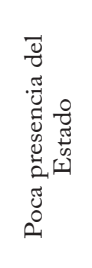 & 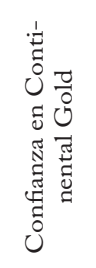 & 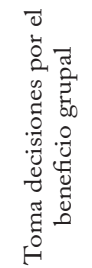 & 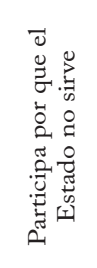 & 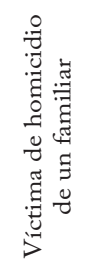 & 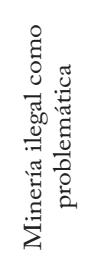 & 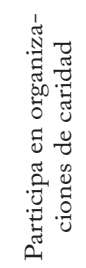 \\
\hline Media & 0,4366 & 0,2958 & 0,1972 & 2,5352 & 0,493 & 0,169 & 0,2113 & 0,2958 & 0,0704 \\
\hline Mediana & 0 & 0 & 0 & 3 & 0 & 0 & 0 & 0 & 0 \\
\hline Moda & 0 & 0 & 0 & 3 & 0 & 0 & 0 & 0 & 0 \\
\hline Desv. est. & 0,4995 & 0,4596 & 0,4007 & 0,9685 & 0,5035 & 0,3774 & 0,4111 & 0,4596 & 0,2577 \\
\hline Mínimo & 0 & 0 & 0 & 1 & 0 & 0 & 0 & 0 & 0 \\
\hline Máximo & 1 & 1 & 1 & 4 & 1 & 1 & 1 & 1 & 1 \\
\hline Observaciones & 71 & 71 & 71 & 71 & 71 & 71 & 71 & 71 & 71 \\
\hline
\end{tabular}

Fuente: elaboración propia. 
De los 31 hombres participantes, 21 eran víctimas de desplazamiento forzado; 14 señalaron que uno de los problemas de Buriticá era la poca presencia del Estado; 35 tomaron sus decisiones en el juego pensando en el beneficio del grupo; 12 dijeron que participaban en organizaciones sociales porque el Estado no sirve; 15 eran víctimas del homicidio de un familiar; 21 señalaron que la minería ilegal era uno de los problemas del municipio y 5 participaban en organizaciones de caridad.

En cuanto a la confianza en la Continental Gold (que no es una variable dicotómica), el promedio reportado fue de 3 (confía algo) en una escala: confía mucho, algo, poco o nada.

\section{MECANISMO DE CONTRIBUCIÓN VOLUNTARIA}

La acción colectiva en Buriticá, representada por los jugadores que invirtieron su dotación inicial en la cuenta del grupo, fue del $42 \%$. Aunque en cada ejercicio hubo valores diferentes (cuadro 7). En el juego con funcionarios públicos locales, el 69\% invirtió en la cuenta del grupo, un valor cercano al 71\% en Vietnam (Cárdenas y Carpenter, 2008), uno de los lugares del mundo de mayor cooperación en el juego MCV.

Cuadro 7

Acción colectiva en Buriticá

(Porcentaje)

\begin{tabular}{ccccc}
\hline Misceláneo & Mineros & Los Asientos & Funcionarios & Buriticá \\
\hline 22 & 42 & 39 & 69 & 42 \\
\hline
\end{tabular}

Fuente: elaboración propia.

Los resultados de los experimentos realizados en Buriticá son 8 puntos porcentuales por debajo del promedio mundial; 20 puntos por encima del promedio latinoamericano; 19 puntos por debajo del promedio del país; 2 puntos por encima de Antioquia y 10 puntos por debajo de otros municipios mineros (cuadro 8).

Cuadro 8

Acción colectiva en otros lugares

(Porcentaje)

\begin{tabular}{cccccc}
\hline $\begin{array}{c}\text { Promedio } \\
\text { Mundial }\end{array}$ & $\begin{array}{c}\text { Promedio } \\
\text { América } \\
\text { Latina }^{2}\end{array}$ & $\begin{array}{c}\text { Promedio } \\
\text { Nacional }^{3}\end{array}$ & $\begin{array}{c}\text { Promedio } \\
\text { Antioquia }^{4}\end{array}$ & $\begin{array}{c}\text { Promedio } \\
\text { Municipios } \\
\text { Mineros }^{5}\end{array}$ & Buriticá \\
\hline 50 & 22 & 61 & 40 & 52 & 42 \\
\hline
\end{tabular}

Fuentes: Cárdenas y Carpenter (2008), Cárdenas, Chong y Ñopo (2008), DNP (2011), Ramírez, Casas, Méndez y Eslava (2013), Eslava (2014). 
Cuadro 9

Acción colectiva en otros territorios mineros del país (Porcentaje)

\begin{tabular}{cccccc}
\hline Tarazá & Segovia & $\begin{array}{c}\text { Puerto } \\
\text { Libertador }\end{array}$ & San Pablo & Cañasgordas & Buriticá \\
\hline 58 & 56 & 54 & 45 & 47 & 42 \\
\hline
\end{tabular}

Fuente: Eslava et al. (2014).

Llama la atención la diferencia negativa de 10 puntos porcentuales frente al agregado de otros municipios mineros donde se ha realizado el juego MCV. El cuadro 9 muestra que, entre los municipios mineros donde se ha aplicado ese método, Buriticá es el que reporta el menor nivel de acción colectiva.

Cuadro 10

Resultado modelos MCO, Logit y Probit

\begin{tabular}{|c|c|c|c|}
\hline Variables independientes & $\mathrm{MCO}$ & Logit & Probit \\
\hline \multirow[t]{2}{*}{ Pocoestado } & $0,291647^{*}$ & $0,463286^{* * * *}$ & $0,4176754^{* *}$ \\
\hline & $(0,1465165)$ & $(0,17605)$ & $(0,17287)$ \\
\hline \multirow[t]{2}{*}{ Ptajeexpect } & $0,0285771^{* * * *}$ & $0,0507795^{* * * *}$ & $0,0461321^{* * * * k}$ \\
\hline & $(0,01051736)$ & $(0,0197144)$ & $(0,0176164)$ \\
\hline \multirow[t]{2}{*}{ Benefgrupo } & $0,1867476^{*}$ & $0,3148596^{*}$ & $0,2829177^{*}$ \\
\hline & $(0,1079279)$ & $(0,16286)$ & $(0,15544)$ \\
\hline \multirow[t]{2}{*}{ Cgold } & $0,1191863^{* *}$ & $0,1823569^{* * *}$ & $0,1743765^{* *}$ \\
\hline & $(0,0510273)$ & $(0,09263)$ & $(0,08715)$ \\
\hline \multirow[t]{2}{*}{ Estadonosirve } & $0,2376862 *$ & $0,4322737^{* * *}$ & $0,4288439^{* * *}$ \\
\hline & $(0,1387478)$ & $(0,19163)$ & $(0,18988)$ \\
\hline \multirow[t]{2}{*}{ Despforzado } & $-0,2015877^{*}$ & $-0,3500249^{* *}$ & $-0,337346^{* *}$ \\
\hline & $(0,1107278)$ & $(0,1512)$ & $(0,14716)$ \\
\hline \multirow[t]{2}{*}{ Decaridad } & $0,3376708^{*}$ & $0,5468749^{* * * *}$ & $0,5447084^{* * * *}$ \\
\hline & $(0,1998114)$ & $(0,17764)$ & $(0,19558)$ \\
\hline \multirow[t]{2}{*}{ Homifamiliar } & 0,1630362 & 0,2805247 & 0,2767507 \\
\hline & $(0.1339474)$ & $(0,21541)$ & $(0,20848)$ \\
\hline \multirow[t]{2}{*}{ Mineriailegal } & $-0,0255917$ & $-0,0153609$ & $-0,0134827$ \\
\hline & $(0,1193202)$ & $(0,17042)$ & $(0,16226)$ \\
\hline _cons & $-1,88024$ & & \\
\hline Observaciones & 71 & 71 & 71 \\
\hline $\mathrm{R}^{2}$ & 0,3932 & & \\
\hline Pseudo $\mathrm{R}^{2}$ & & 0,37118935 & 0,37182002 \\
\hline
\end{tabular}

Nota: Para el modelo MCO se muestran los coeficientes estimados y los errores robustos. Para los modelos logit y probit se muestran los efectos marginales.

* Significativo al $10 \%$. ${ }^{* *}$ significativo al $5 \%$. ${ }^{* * *}$ significativo al $1 \%$.

Fuente: elaboración propia.

\section{RESULTADOS}

El bajo número de participantes limitó la inclusión de variables en los modelos. Para mantener un pseudo $\mathrm{R}^{2}$ alto, se incluyeron 9 independientes fueron y 7 de ellas resultaron estadísticamente significativas y con el respaldo teórico de ser determinantes de la acción colectiva. Estas variables se incluyeron en modelos MCO, Logit y Probit. E1 modelo MCO se descartó porque la varianza del error no era cons- 
tante, pues es función de X, y así no es homocedástico. Además, tiene la restricción de que los valores estimados de respuesta estén entre 0 y 1 (Moscote y Rincón, 2012).

Para elegir entre el logit y el probit se analizaron sus pseudo $\mathrm{R}^{2}$, cuya diferencia es marginal (Logit $=0,3711$ y Probit $=0,3718$ ). Se eligió el Probit, que predice correctamente que el 77,5\% de las personas aportaron efectivamente a la cuenta del grupo. Además, la curva ROC - una medida de bondad del ajuste que va de 0,5 a 1- cubre un área de 0.8829 .

Gráfica 2

Curva ROC del modelo probit

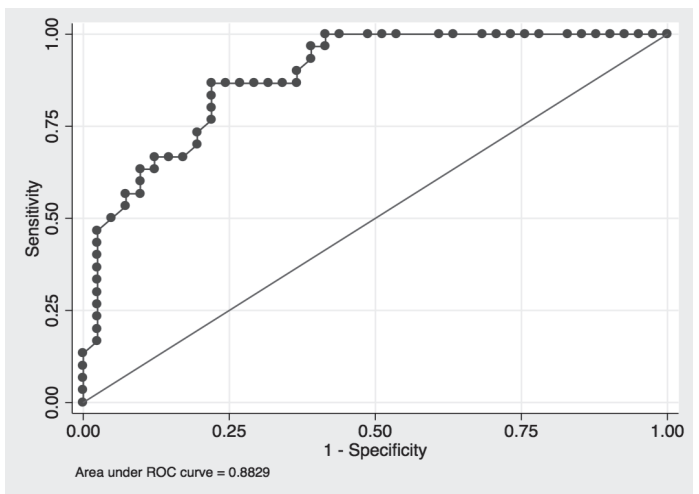

Fuente: elaboración propia.

\section{MODELO ESTIMADO}

Los resultados del modelo seleccionado indican el signo y la magnitud de los efectos marginales de los determinantes. Y confirman la relación entre acción colectiva y los que examinamos a continuación.

\section{ESTADO LOCAL}

Según la literatura, cuando el orden administrativo y político no es coherente con el orden social, étnico y comunitario, la disposición a cooperar es menor. La variable 'pocoestado', resultante de la pregunta ¿cuáles son los tres problemas prioritarios en Buriticá?, indica que los jugadores que contestan poca presencia estatal tienen una probabilidad 42 puntos porcentuales mayor de aportar a la cuenta del grupo. Esto es consistente con la tesis de que el mal funcionamiento del Estado o su escasa presencia (como en Buriticá) llevan a que las personas 
Cuadro 11

Resultados del modelo Probit

\begin{tabular}{ll}
\hline Variables independientes & Probit \\
\hline Pocoestado & $\frac{0,4176754^{* * *}}{(0,17287)}$ \\
\hline Ptajeexpect & $\frac{0,0461321^{* * *}}{(0,0176164)}$ \\
\hline Benefgrupo & $\frac{0,2829177^{*}}{(0,15544)}$ \\
\hline Cgold & $\frac{0,1743765^{* *}}{(0,08715)}$ \\
\hline Estadonosirve & $\frac{0,4288439^{* * *}}{(0,18988)}$ \\
\hline Despforzado & $\frac{-0,337346^{* *}}{(0,14716)}$ \\
\hline Decaridad & $\frac{0,5447084^{* * *}}{(0,19558)}$ \\
\hline Homifamiliar & $\frac{0,2767507}{(0,20848)}$ \\
\hline Mineriailegal & $\frac{-0,0134827}{(0,16226)}$ \\
\hline Observaciones & 71 \\
\hline Pseudo R ${ }^{2}$ & 0,37182002 \\
\hline
\end{tabular}

*Estadísticamente significativo al 10\%. ** estadísticamente significativo al 5\%. ***estadísticamente significativo al $1 \%$.

Fuente: elaboración propia.

recurran a arreglos endógenos y cooperen para encontrar soluciones más eficientes y sostenibles (Dippel, 2014).

Una segunda variable estimada a este respecto fue "estadonosirve”, que se construyó a partir de la encuesta postjuego mediante la pregunta: entre las siguientes opciones, ¿cuál es la principal razón que lo motiva a participar en el grupo u organización? Siguiendo la lógica anterior, las personas motivadas a participar en una organización porque el Estado no sirve tienen una probabilidad 43 puntos porcentuales mayor de invertir en la cuenta del grupo. El modelo también estima el efecto de la variable 'mineríailegal', que tuvo un efecto marginal negativo pero un valor $\mathrm{p}$ mayor que 0,1 ; de modo que no resultó significativa.

\section{AVERSIÓN AL RIESGO Y EXPECTATIVAS}

La variable 'ptajeexpect' corresponde a los resultados de preguntar en el MCV ¿cuántas personas cree usted que van a invertir en la cuenta del grupo? Por cada persona en promedio que el grupo cree que va a aportar a la cuenta del grupo, hay una probabilidad 4 puntos porcentuales mayor de aportar a esa cuenta. Esto obedece a que el comportamiento de grupo genera presión para el cumplimiento y reduce la incertidumbre y el riesgo de pérdidas. 
La variable 'benefgrupo', construida a partir de las respuesta a la pregunta ¿qué motivó las decisiones en los juegos?, indica que las personas que toman decisiones en beneficio del grupo, tienen una probabilidad 28 puntos porcentuales mayor de aportar a la cuenta del grupo. En vez de conservar sus $\$ 15.000$ iniciales y ganar $\$ 1.500$ por cada aportante a la cuenta del grupo, aportan los $\$ 15.000$ a esa cuenta y cooperan para el beneficio del grupo.

\section{VICTIMIZACIÓN}

Este determinante, representado por 'despforzado', se deriva de la pregunta ¿ha sido víctima de algunos de los siguientes delitos? Según la literatura, la victimización es un detonante de la participación. En los juegos experimentales realizado por Eslava et al. (2014) se encontró que la victimización por desplazamiento forzado aumentó en casi 22 puntos porcentuales la probabilidad de aportar a la cuenta del grupo.

Nuestros resultados indican una relación inversa entre acción colectiva y victimización por desplazamiento. En promedio, las víctimas por desplazamiento forzado tienen una probabilidad 33 puntos porcentuales menor de aportar a la cuenta del grupo que los demás jugadores. Cabe señalar que los mecanismos de respuesta a la victimización por desplazamiento forzado son diferentes según el contexto. En algunos casos llevan a que la víctima construya nuevos vínculos sociales y en otros a una menor disposición a la acción colectiva.

Con respecto a este determinante, también se estimó el efecto de la variable 'homifamiliar', pero su valor $\mathrm{p}$ fue mayor que 0,1 , de modo que no resultó significativa.

\section{FAMILIARIDAD Y CONFIANZA}

Este determinante se deriva de las respuestas a la pregunta ¿Participa en alguno de los siguientes grupos u organizaciones? Según la literatura, la asociación en grupos a nivel micro refuerza el capital social: los atributos de comunidad. En nuestro caso encontramos que quienes participan en organizaciones de caridad, tienen una probabilidad 54 puntos porcentuales mayor de invertir en la cuenta del grupo. Como viven en un entorno de prosocialidad, conocen los beneficios de la acción en grupo.

Por último, la variable 'cgold' representa la confianza en la Continental Gold. Esta se construyó mediante la pregunta ¿cuánto confía en las siguientes instituciones? Y se podía dar una sola respuesta de las siguientes opciones: poco, algo o mucho. $\mathrm{El}$ resultado es que por 
cada escalafón positivo que se selecciona en referencia a la confianza en Continental Gold (siendo la respuesta 'poco' la más negativa y 'mucho' la más positiva), los jugadores tienen 17 puntos porcentuales más de probabilidad de contribuir a la cuenta grupal.

\section{LECCIONES}

\section{VACÍOS ESTATALES}

En Buriticá, como en otras zonas rurales del país, hay una débil presencia del Estado, si la hay. Cuando el Estado aumenta su acción en un territorio, y no lo hace bien, menoscaba los atributos comunitarios locales, bien sea porque eleva los costos de transacción, porque la supervisión a cargo de una autoridad externa desincentiva la supervisión horizontal, o por otras razones.

Por ello es importante el diseño experimental y su validez externa en el sentido de Smith. El estudio de los asuntos públicos considerando la acción colectiva y los atributos de comunidad aporta muchos insumos, y sobre todo más y mejor información para la toma de decisiones y el diseño de políticas.

Además de ocuparse del despliegue territorial, el Estado debe preocuparse por la manera de ejercer su autoridad y por el capital social inherente a la comunidad. Es decir, no debe sólo buscar un mayor gobierno sino también una mejor gobernanza.

\section{CROWDING-OUT}

Es llamativo que entre los municipios mineros de los que se obtuvo información mediante el ejercicio MCV, Buriticá es el que muestra el menor nivel de cooperación. En este punto es preciso mencionar el menoscabo y la erosión de la cooperación en la comunidad como consecuencia de diseños estatales y mercantiles desligados de la realidad colectiva (Cárdenas y Eslava, 2017), que en la literatura se denomina crowding out.

La llegada de la Continental Gold coincide con un fenómeno de menoscabo institucional (institutional crowding out) ${ }^{3}$, como muestran los datos experimentales. Esta erosión se podría atenuar si la compañía emprende la tarea de conocer los atributos de la comunidad buritiqueña y realiza acciones de responsabilidad social que articulen a los tres actores de la gobernanza-Estado, comunidad y mercado-y

${ }^{3}$ Cárdenas (2009). El término institutional crowding out alude a la erosión de las preferencias prosociales (Eslava, 2017).

Revista de Economía Institucional, vol. 22, N.o 42, primer semestre/2020, pp. $269-295$ ISSN OI $24-5996 / E-I S S N ~ 2346-245^{\circ}$ 
fortalezcan el comportamiento prosocial, para restablecer los lazos de confianza y lograr una relación armónica y cooperativa.

\section{CONFIANZA}

También resultaron relevantes algunas variables como participar en una organización de caridad y tomar decisiones en beneficio del grupo. Esto indica que así la comunidad viva en un entorno donde el Estado es débil y se expande la minería, se conservan disposiciones y atributos para la cooperación. Al municipio no solo ha llegado la Continental Gold sino también forasteros dedicados a la actividad minera. Según testimonios de la comunidad, esa irrupción ha tenido varios efectos, como el alza inusitada de precios los alimentos y alquileres, un boom poblacional y la intervención estatal mediante acciones militares.

Todo esto ha debilitado la confianza en el Estado y en la compañía, que al excluir a la comunidad en su proceso de toma de decisiones la han expuesto a situaciones de incertidumbre y de riesgo, reduciendo su disposición a cooperar. Para superar esta situación, el Estado y el sector privado deberían valorar el capital social existente, frenar su desgaste $\mathrm{y}$ evitar que sus acciones lo desincentiven en vez de promoverlo.

\section{LA VIDA COTIDIANA Y EL SENTIDO COMÚN}

"En las ciencias sociales se observa una sobrevaloración de las herramientas cuantitativas" (González, 2005). Se cree en vano que estas ofrecen una representación precisa del mundo y de los fenómenos estudiados. No obstante, sí permiten construir relatos a partir de hechos, por ejemplo, de la tendencia a cooperar en una comunidad. Pero la magnitud de la cooperación solo es un número, cuya comprensión requiere un contexto o realidad novelada.

El ejercicio de levantar la base de datos, diseñar y realizar los experimentos, conocer el municipio y reconocer sus atributos permitió construir e interpretar los datos en su contexto. Además, hizo posible explicar los hallazgos no solo a la luz de la técnica sino también de las vivencias en el lugar. Es decir, acercarse a la vida cotidiana, que en últimas es la que revela el sentido común de las personas, que difieren entre sí, y no son un agente representativo (Lechner, 1988).

Siguiendo a Juan Camilo Cárdenas, uno de los pioneros del método experimental en el país, los experimentos permiten acercarse a la riqueza de la realidad, y entender mejor la interacción entre teoría, observación y cambio social. La investigación que conecta estos tres aspectos permite identificar y entender los dilemas sociales, hacer 
recomendaciones basadas en el contexto y "susurrar al oído de los príncipes" (Méndez, 2013).

Y, en opinión de Adolfo Eslava, los experimentos -como fuente de conocimiento- permiten establecer la triada políticos-técnicoscomunidades como escenario para construir y retroalimentar el juego de políticas públicas. El presente ejercicio busca ayudar a superar el desatino en el estudio de la vida cotidiana: las "distancias entre conocimiento y conciencia, entre ciencia y sentido común" (Lechner, 1988, p. 51).

\section{REFERENCIAS BIBLIOGRÁFICAS}

Aguilar, L. F. (2013). El estudio de las políticas públicas. México D.F: Porrúa.

Andreoni, J. (1995). Cooperation in public-goods experiments: kindness or confusion? American Economic Review, 85(4), 891-904.

Attanasio, O., Espinosa, M. et al. (2011). Seguimiento de la evaluación de impacto de los programas paz y desarrollo y Laboratorios de Paz. Bogotá: DNP.

Blume, A. y Board, O. (2013). Language barriers. Econometrica, 81(2), 781-812.

Bowles, S. (2010). Microeconomía comportamiento, instituciones y evolución. Bogotá: Universidad de los Andes.

Bowles, S. y Gintis, H. (2002). Social capital and community governance. Economic Journal, 112(483), F419-F436

Bowles, S. y Gintis, H. (2005). Social capital, moral sentiments, and community governance. En H. Gintis y S. Bowles (eds.). Moral sentiments and material interests (pp. 379-398). Londres: MIT Press.

Caballero, K., Espitia, J. E. et al. (2017). Minería en Colombia: Institucionalidad y territorio, paradojas y conflictos. Bogotá: Contraloría General de la República.

Candelo, N. y Polanía, S. (2008). Pasos metodológicos de un diseño experimental para medir capital social y acción colectiva en seis ciudades latinoamericanas. Documentos CEDE, 005104. Bogotá: Universidad de los Andes.

Cárdenas, J. C. (2009). Dilemas de lo colectivo. Instituciones, pobreza y cooperación en el manejo local de los recursos de uso común. Bogotá: Universidad de los Andes.

Cárdenas, J. C. y Carpenter, J. (2008). Behavioral development economics: Lessons from field labs in the developing world. Journal of Development Studies, 44(3), 311-338.

Cárdenas, J. C., Chong, A. et al. (2008). Stated social behavior and revealed actions: Evidence from six Latin American countries using representative samples. Development Bank, Research Department working paper 634.

Cárdenas, J. C., Maya, D. L. et al. (2003). Métodos experimentales y participativos para el análisis de la acción colectiva y la cooperación en el uso de recursos naturales por parte de comunidades rurales. Cuadernos de desarrollo rural, 50, 63-96. 
Cárdenas, J. C. y Ostrom, E. (2004). What do people bring into the game? Experiments in the field about cooperation in the commons. Agricultural systems, 82(3), 307-326.

Cardenas, J. C., Stranlund, J. et al. (2000). Local environmental control and institutional crowding-out. World development, 28(10), 1719-1733.

Cardona, A. y Cuadros, M. A. (2014). Minería y patrimonio ambiental en Buriticá. Medellín: Conciudadanía.

Casas, A., Giraldo, J. et al. (2013). Valores, representaciones y capital social en Antioquia. Medellín: Eafit-Gobernación de Antioquia.

Casas, A. C. y Méndez, N. M. (2013). Experimentos en ciencias sociales: usos, métodos y aplicaciones. Bogotá: Pontificia Universidad Javeriana.

Coleman, E. y Ostrom, E. (2011). Experimental contributions to collective action theory. En J. N. Druckman., D. P. Green. et al (eds.), Cambridge handbook of experimental political science (pp. 635-661). Nueva York: Cambridge University Press.

Continental Gold. (2017). Informe de sostenibilidad año 2016.

Departamento Administrativo Nacional de Estadística, [www.dane.gov.co].

Dippel, C. (2014). Forced coexistence and economic development: evidence from Native American Reservations. Econometrica, 82(6), 2131-2165.

Elster, J. (2009). Tuercas y tornillos. Bogotá: Gedisa.

Eslava, A. (2017). Politicos, técnicos y comunidades. Medellín: Universidad Eafit.

Eslava, A. y Preciado, A. F. (2014). ¿Primero yo, después los míos y de últimos los otros? Confianza y acción colectiva: retos y políticas públicas. Revista Facultad de Derecho y Ciencias Politicas, 44(121), 577-607.

Eslava, A. et al. (2014). Oro como fortuna. Instituciones, capital social y gobernanza de la minería aurífera colombiana. Medellín: Universidad Eafit-Colciencias.

Frey, B. S. y Meier, S. (2004). Social comparisons and pro-social behavior: Testing "conditional cooperation" in a field experiment. American Economic Review, 94(5), 1717-1722.

Giraldo, J. et al. (2013). Valores, representaciones y capital social en Antioquia. Medellín: Universidad Eafit.

González, J. I. (2005). La economía y el dato novelado. En C. Hernández y J. López, Navegaciones. El magisterio y la investigación (pp. 174-187). Bogotá: Colciencias-Unesco.

Hardin, G. (1968). The tragedy of the commons. Science, 13(162), 12431248.

Jackson, M. O., Rodríguez-B., T. et al. (2012). Social capital and social quilts: Network patterns of favor exchange. American Economic Review, 102(5), 1857-1897.

Lechner, N. (1988). Patios interiores de la democracia. Santiago de Chile: Flacso.

Ledyard, J. O. (1995). Public goods: A survey of experimental research. En J. Kagel y A. Roth (eds.), Handbook of Experimental Economics (pp. 111-194). Princenton: Princenton University Press.

Levitt, S. D. y List, J. A. (2007a). On the generalizability of lab behaviour to the field. Canadian Journal of Economics/Revue canadienne d'économique, 40(2), 347-370. 
Levitt, S. D. y List, J. A. (2007b). What do laboratory experiments measuring social preferences reveal about the real world? Journal of Economic perspectives, 21(2), 153-174.

Marwell, G. y Ames, R. E. (1981). Economists free ride, does anyone else? Experiments on the provision of public goods, IV. Journal of public economics, 15(3), 295-310.

Méndez M., N. (2012). Una propuesta metodológica para la medición de capital social en victimas del conflicto armado. Tesis Doctoral. Bogotá: Uniandes.

Méndez M., N. (2013). La esencia metodológica del diseño de experimentos para la investigación en ciencias sociales. En Experimentos en ciencias sociales: usos, métodos y aplicaciones (pp. 25- 52). Bogotá: Pontificia Universidad Javeriana.

Morton, R. B. y Williams, K. C. (2008). Experimentation in political science. En B. Morton y K. Williams (ed.), The Oxford handbook of political methodology (pp. 339-356). Oxford: University Press.

Morton, R. B. y Williams, K. C. (2010). Experimental political science and the study of causality: From nature to the lab. Cambridge: Cambridge University Press.

Morton, R. B. (2013). Prólogo. En A. C. Casas, y N. M. Méndez, Experimentos en ciencias sociales: usos, métodos y aplicaciones (pp. 11-13). Bogotá: Pontificia Universidad Javeriana.

Moscote, O. y Rincón, W. A. (2012). Modelo logit y probit: un caso de aplicación. Comunicaciones en Estadística, 5(2), 123-133.

North, D. (1993). Instituciones, cambio institucional y desempeño económico. México DF: Fondo de Cultura Económica.

Olson, M. (1965). The logic of collective action. Cambrige, Mass.: Harvard University Press.

Ostrom, E. (1990). Governing the commons. Cambridge: Cambridge University Press.

Ostrom, E. (2013). Más allá de los mercados y los Estados: gobernanza policéntrica de sistemas económicos complejos. Revista Mexicana de Sociología, 76(SPE), 15-70.

Ostrom, E. y Ahn, T. K. (2003). A social science perspective on social capital: Social capital and collective action. Revista Mexicana de Sociología, 65(1), 155-233.

Pérez. O. I. E1 sector minero energético en Colombia. Importancia macroeconómica y transformaciones recientes. En L. Valencia y A. Riaño. La minería en el posconflicto. Un asunto de quilates (pp. 54-93). Bogotá: Ediciones B.

Plott, C. R. y Smith, V. L. (2008). Handbook of experimental economics results. Ámsterdam: Elsevier.

Rettberg, A., Cárdenas, J. C. et al. (2017). Mismo recurso, diferentes conflictos: Un análisis de la relación entre oro, conflicto y criminalidad en seis departamentos colombianos. Documentos CEDE, 64, 1-56.

Rudas, G. y Espitia, J. E. (2013). Participación del estado y la sociedad en la renta minera. En L. J. Garay (ed.), Minería en Colombia. Fundamentos para superar el modelo extractivista (pp. 125-172). Bogotá: Contraloría General de la República. 
Rudas, G. y Zamora, J. (2013). La paradoja de la minería y el desarrollo. Análisis departamental y municipal para el caso de Colombia. En L. J. Garay (ed.), Minería en Colombia. Institucionalidad y territorio, paradojas y conflictos (pp. 27-76). Bogotá: Contraloría General de la República.

Saijo, T. (2008). Spiteful behavior in voluntary contribution mechanism experiments. En C. R. Plott, y V. L. Smith. Handbook of experimental economics results (pp. 802-816). Ámsterdam: Elsevier.

Saijo, T. y Nakamura, H. (1995). The "spite" dilemma in voluntary contribution mechanism experiments. Journal of Conflict Resolution, 39(3), 535-560.

Schram, A. (2005). Artificiality: The tension between internal and external validity in economic experiments. Journal of Economic Methodology, 12(2), 225-237.

Smith, V. L. (1982). Microeconomic systems as an experimental science. American Economic Review, 72(5), 923-955.

Subirats, J. (2010). Si la respuesta es gobernanza, ¿cuál es la pregunta? Factores de cambio en la política y en las políticas. EKONOMLAZ. Revista Vasca de Economía, 74(02), 16-35.

Tilly, C. y Wood, L. J. (2009). Los movimientos sociales 1768-2008. Desde sus orígenes hasta Facebook. Sociológica, 29(81), 295-300.

Valencia, L. y Riaño, A. (2017). La minería en el posconflicto. Un asunto de quilates. Bogotá: Ediciones $\mathrm{B}$.

Voors, M. J. et al. (2012). Violent conflict and behavior: A field experiment in Burundi. American Economic Review, 102(2), 941-964.

Wolitzky, A. (2012). Cooperation with network monitoring. Review of Economic Studies, 80(1), 395-427.

Wooldridge, J. M. (2010). Econometric analysis of cross section and panel data. Cambridge, Mass.: MIT Press. 